\title{
Morfología Sagital del Raquis en Palistas Jóvenes de Alto Nivel
}

\author{
Sagittal Spinal Morphology in Young Highly-Trained Paddlers
}

"José M. Muyor; **Fernando Alacid \& **** Pedro A. López-Miñarro

MUYOR, J. M.; ALACID, F.; LÓPEZ-MIÑARRO, P. A. Morfología sagital del raquis en palistas jóvenes de alto nivel. Int. J. Morphol., 29(3):1047-1053, 2011.

RESUMEN: El objetivo fue valorar la morfología raquídea del raquis torácico y lumbar en piragüistas de la modalidad de kayak de categoría cadete. A un total de 66 kayakistas de aguas tranquilas, 36 varones y 30 mujeres de categoría cadete (media de edad 16,22 $\pm 0,80$ años), se les evaluó mediante un Spinal Mouse la morfología sagital del raquis torácico y lumbar en bipedestación, decúbito prono y en flexión máxima del tronco en sedentación con rodillas extendidas (test sit-and-reach). La cifosis torácica en los varones fue superior en las tres posiciones analizadas, especialmente en el test sit-and-reach. El porcentaje de casos con hipercifosis torácica fue de un $42,1 \%$ en los varones y un 21,4\% en las mujeres. Los valores angulares en decúbito prono fueron inferiores que en bipedestación (p < 0,001). Aquellos deportistas con hipercifosis torácica tenían un valor angular menor en decúbito prono $\left(37,85 \pm 9,42^{\circ}\right)$ que en bipedestación $\left(50,95 \pm 5,12^{\circ}\right)$. En la curva lumbar no se encontraron diferencias entre géneros en las posiciones evaluadas. En el raquis lumbar, predominaron las angulaciones consideradas normales (81,6\% en varones y 75\% en mujeres). En conclusión, la morfología sagital del raquis en kayakistas de categoría cadete se caracteriza por una tendencia a la hipercifosis torácica, especialmente en los varones, si bien se trata de curvas no estructuradas. En cuanto a la curva lumbar en bipedestación presenta un alto porcentaje de ángulos considerados normales. La práctica del piragüismo/canotaje de aguas tranquilas en su modalidad de kayak genera una mayor capacidad de flexión intervertebral lumbar, que produce un alto porcentaje de morfotipos cifóticos. Es preciso adoptar medidas de intervención postural para mejorar la morfología raquídea de los palistas de aguas tranquilas.

PALABRAS CLAVE: Postura; Curvaturas sagitales; Columna vertebral; Deporte.

\section{INTRODUCCIÓN}

La morfología del raquis en el plano sagital se caracteriza por la presencia de unas curvaturas con un intervalo de grados considerados normales (Kapandji, 1980). En algunos casos, estas curvas aumentan o disminuyen su magnitud dando lugar a desalineaciones raquídeas (Tüzün et al., 1999). La evolución de la morfología sagital del raquis está influida por diversos factores. La edad y el género condicionan su morfología, así como otros factores que pueden derivar en alteraciones que aumentan el riesgo de algias vertebrales (Cil et al., 2005; Masharawi et al., 2010).

La práctica deportiva intensa también se ha relacionado con determinadas modificaciones en la morfología sagital del raquis. Las posturas y gestos específicos de los entrenamientos parecen ser factores que inciden en las adaptaciones raquídeas, debido a la incidencia que tienen las cargas mecánicas sobre los tejidos óseos (Wojtys et al., 2000).

Diversos estudios han observado adaptaciones raquídeas específicas en diferentes deportes. En aquellos con un predominio de posiciones en flexión del tronco se ha encontrado un alto porcentaje de posturas hipercifóticas (Alricsson \& Werner, 2006; Rajabi et al., 2007; LópezMiñarro et al., 2008; Föster et al., 2009), mientras que en deportes caracterizados por posiciones de extensión y alineación de la columna vertebral, como en danza y en gimnasia rítmica, se ha mostrado una disminución de la cifosis torácica, reduciéndose el porcentaje de hipercifosis torácica (Nilsson et al., 2003; Kums et al., 2007). Habitualmente, el

* Facultad de Educación. Universidad de Almería, España.

** Facultad de Ciencias del Deporte. Universidad de Murcia, España.

**** Facultad de Educación. Universidad de Murcia, España.

Este estudio ha sido financiado con una ayuda de la Fundación Séneca-Agencia de Ciencia y Tecnología de la Región de Murcia (II PCTRM 2007-2010) con nº 11951/PI/09 (Evolución de las curvaturas sagitales del raquis, extensibilidad isquiosural, dolor lumbar y características antropométricas de piragüistas de élite). 
análisis de la morfología del raquis se ha centrado en la postura de bipedestación, si bien también es importante realizar una valoración en flexión del tronco para detectar desalineaciones "dinámicas" que no son detectables en bipedestación.

El piragüismo/canotaje, en su modalidad de kayak, tiene una gran implicación de la columna vertebral. La actividad del kayakista se basa en una sedentación prolongada con rodillas prácticamente extendidas, y un paleo que exige una gran implicación del raquis en el plano transversal. Fernández et al. (1992) indicaron que pueden existir alteraciones de la morfología del raquis en palistas, tales como hipercifosis torácica e hiperlordosis lumbar. Estudios previos han analizado la morfología raquídea en palistas de categoría infantil encontrando una tendencia a posturas hipercifóticas (López-Miñarro et al., 2009; LópezMiñarro \& Alacid, 2010). No obstante, puesto que la postura se modifica con el proceso de maduración vertebral, y el volumen de entrenamiento se ha identificado como una variable que incide en las adaptaciones de las curvas del raquis (Wojtys et al.), es necesario valorar las características morfológicas del raquis en piragüistas de otras categorías.

La importancia de detectar alteraciones sagitales en la morfología raquídea está basada en las repercusiones asociadas a ellas. Diversos estudios han establecido que las posturas de mayor cifosis torácica se relacionan con un mayor nivel de estrés compresivo y de cizalla anteroposterior (Keller et al., 2005; Brigss et al., 2007), y una mayor frecuencia de algias vertebrales (Smith et al., 2008). Además, las posturas de mayor flexión intervertebral se han asociado con un aumento de la presión intradiscal (Wilke et al., 1999; Polga et al., 2004).

Conocer la evolución de la morfología del raquis en deportistas así como la influencia de la práctica intensa de un deporte en la morfología raquídea es importante para implementar las medidas terapéuticas y las adaptaciones en el entrenamiento más adecuadas. Puesto que diversos estudios han encontrado una relación entre los movimientos repetitivos y posturas específicas del deporte con determinadas adaptaciones en la morfología del raquis, el objetivo del presente estudio fue analizar la morfología raquídea del raquis torácico, lumbar e inclinación pélvica en kayakistas de categoría cadete.

\section{MATERIAL Y MÉTODO}

Un total de 66 piragüistas de categoría cadete (15-16 años) (media de edad 16,22 $\pm 0,80$ años), participaron voluntariamente en este estudio (Tabla I). Los criterios de inclusión fueron: un volumen de entrenamiento de 2 a 3 horas al día, con una frecuencia de 4 a 6 días por semana, y un historial de entrenamiento en piragüismo/canotaje de más de 5 años de práctica ininterrumpida. Los criterios de exclusión fueron: 1) haber manifestado dolor lumbar en los tres meses anteriores a la realización del estudio; 2) haber sido operado de la columna vertebral o miembros inferiores; y 3 ) tener dolor lumbar o torácico en el momento de las valoraciones.

Procedimientos. El estudio fue aprobado por el Comité Ético y de Investigación de la Universidad de Murcia. Previamente a las mediciones, todos los sujetos y sus tutores legales, así como sus entrenadores, fueron informados sobre los procedimientos del estudio y firmaron, voluntariamente, un consentimiento informado.

Antes de iniciar las mediciones, el investigador principal identificó mediante palpación y marcó, con un lápiz dérmico, la apófisis espinosa de la séptima vértebra torácica (C7), así como la tercera vértebra sacra (S3). A continuación, la morfología de la curva torácica y lumbar fueron valoradas mediante un Spinal Mouse ${ }^{\circledR}$ (Idiag, Suiza), en un orden aleatorio, en las posiciones de bipedestación, flexión máxima del tronco con rodillas extendidas (test sit-andreach) y decúbito prono. Entre cada medición hubo 5 minutos de descanso. Cada sujeto fue evaluado por el mismo examinador en una misma sesión de valoración. La temperatura del laboratorio donde se realizaron las mediciones fue estandarizada a $24^{\circ} \mathrm{C}$.

Para medir la morfología raquídea, una vez que el sujeto se colocaba en la posición a medir, se situaba el Spinal Mouse en la marca de C7 y se desplazaba en sentido cráneo-caudal a lo largo de las apófisis espinosas del raquis, hasta la marca de S3 (Fig. 1). A continuación, el software del sistema digitalizaba el contorno del raquis en el plano sagital, aportando información sobre la angulación global de las curvas raquídeas (Fig. 2). En cuanto a la curva lumbar, valores negativos correspondieron a una curva de concavidad posterior (lordosis), y los valores positivos a una curva de concavidad anterior (cifosis lumbar).

Tabla I. Características de la muestra. * p <0,001 respecto a las mujeres.

\begin{tabular}{lcccc}
\hline Género & $\mathbf{n}$ & Edad (años) & Talla $(\mathbf{c m})$ & Masa $(\mathbf{k g})$ \\
\hline Varones & 36 & $16,31 \pm 0,41$ & $175,29 \pm 6,15^{*}$ & $70,82 \pm 8,63^{*}$ \\
Mujeres & 30 & $15,93 \pm 1,01$ & $164,01 \pm 4,78$ & $60,41 \pm 5,90$ \\
\hline
\end{tabular}




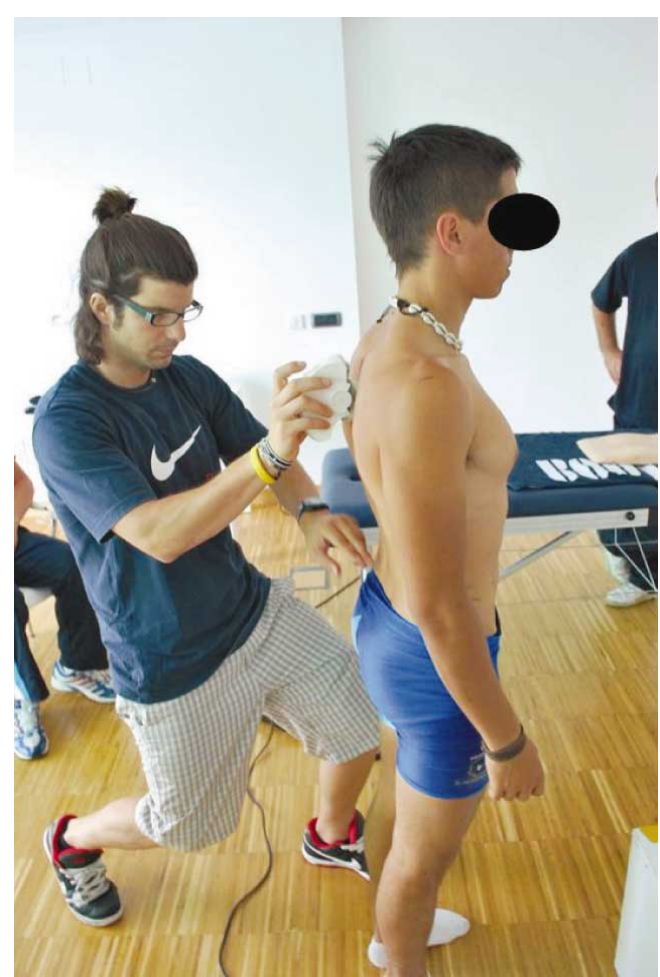

Figura 1. Valoración del raquis y pelvis en bipedestación mediante el Spinal Mouse ${ }^{\circledR}$.

\section{Posturas}

Decúbito prono. Se solicitó a los deportistas que se colocaran en una esterilla en decúbito prono, colocando los brazos en los costados y la frente apoyada en el suelo.

Bipedestación. Los sujetos se situaban de pie, con los hombros relajados, mirada al frente, los brazos a lo largo del tronco y con una apertura de los pies igual a la anchura de sus caderas. Para categorizar los valores angulares de la curva torácica en bipedestación y en decúbito prono en base a unas referencias de normalidad, se utilizó la propuesta de Mejia et al. (1996): cifosis torácica normal (entre $20^{\circ}$ y $45^{\circ}$ ), rectificación o hipolordosis torácica $\left(<20^{\circ}\right)$, e hipercifosis torácica $\left(>45^{\circ}\right)$. Respecto a la curva lumbar, valores entre $20^{\circ}$ y $40^{\circ}$ fueron considerados como lordosis normal, mientras que valores inferiores a $20^{\circ}$ se consideraron como hipolordosis y valores superiores a $40^{\circ}$ como hiperlordosis (Tüzün et al.).

Flexión máxima del tronco en sedentación con rodillas extendidas (test sit-and-reach). Para realizar el test, el deportista se situó en sedentación, con las rodillas extendidas y los pies separados a la anchura de sus caderas. Las plantas de los pies se colocaron perpendiculares al suelo, en contacto con el cajón de medición. En esta posición el deportista realizó una flexión máxima del tronco con rodillas y brazos extendidos. Las palmas de las manos, una sobre la otra, se tenían que deslizar

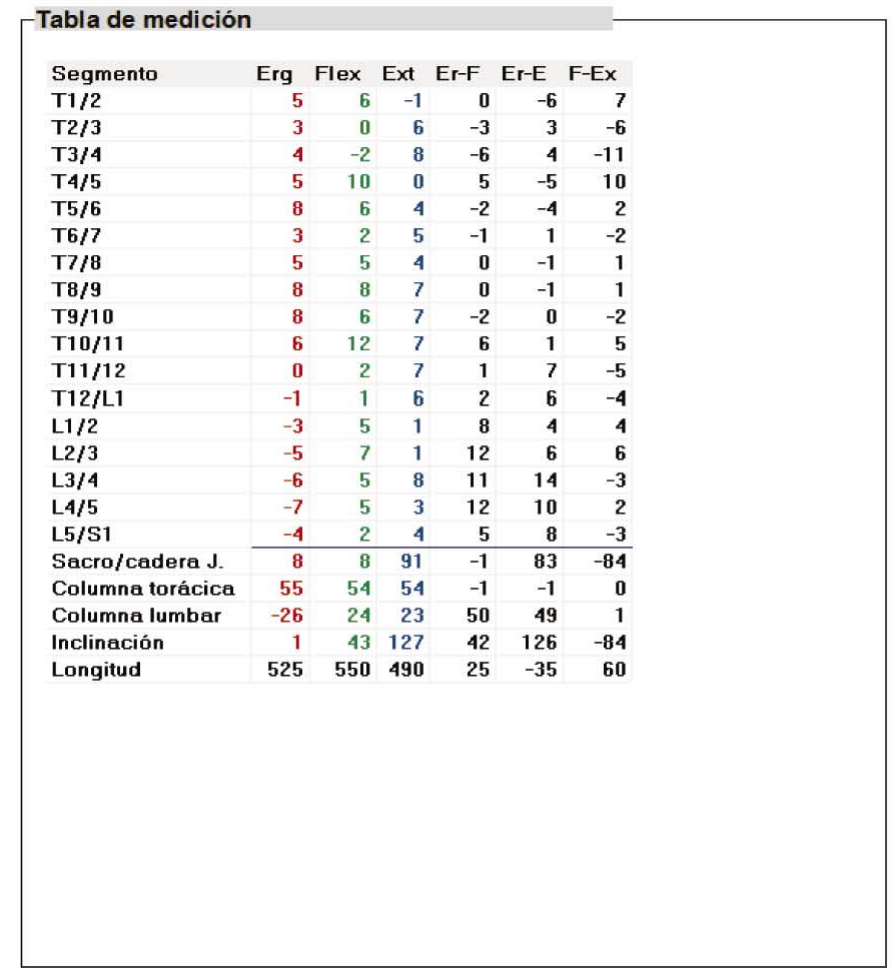

Fig. 2. Información aportada por el Spinal Mouse®.

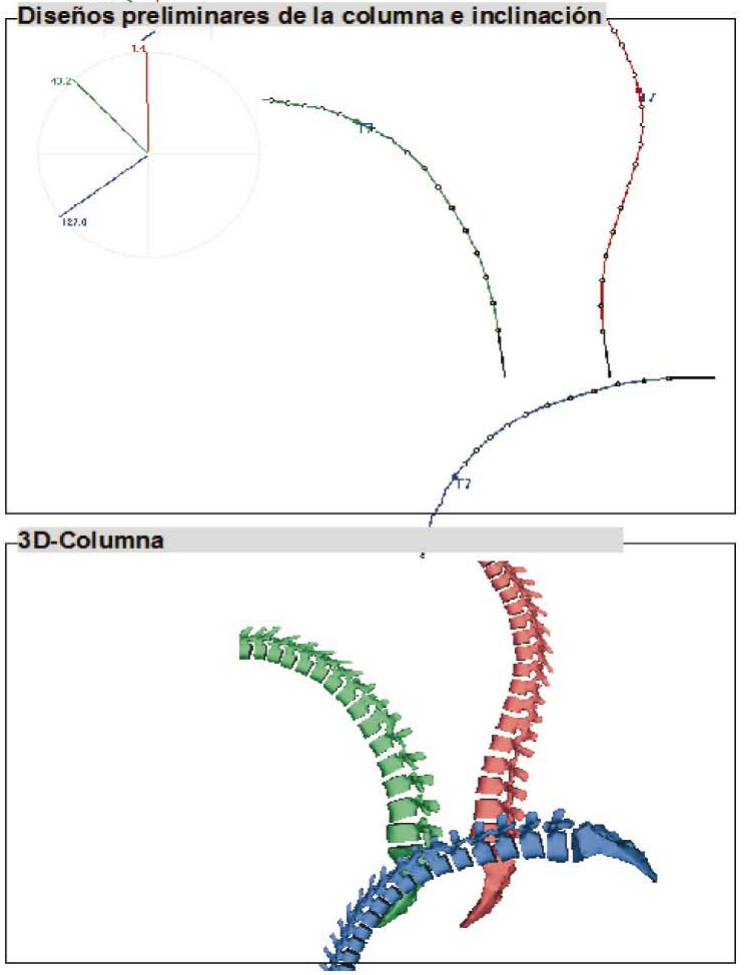


sobre el cajón, hasta alcanzar la máxima distancia posible, manteniendo la posición durante 5 segundos. Al alcanzar la máxima distancia, se procedía a la medición de la morfología del raquis y pelvis mediante el Spinal Mouse. Para clasificar los valores angulares del raquis en el test sit-and-reach se utilizaron las referencias aportadas por Pastor (2000), que estableció, para la curva torácica, valores normales cuando eran inferiores a $67^{\circ}$, morfotipo cifótico leve entre 67 y $76^{\circ}$, y morfotipo cifótico moderado aquellos valores superiores a $76^{\circ}$. En cuanto a la curva lumbar, valores inferiores a $23^{\circ}$ fueron considerados normales, mientras que valores entre 23 y $31^{\circ}$ correspondieron a un morfotipo cifótico leve, y superiores a $31^{\circ}$ un morfotipo cifótico moderado.

Análisis estadístico. Se realizó un análisis descriptivo de cada una de las variables, obteniendo sus valores medios y desviaciones típicas. Tras aplicar el test de normalidad de Kolmogorov-Smirnov y comprobar que las variables seguían una distribución normal, y para comparar la morfología raquídea entre las diferentes posiciones analizadas, se realizó un análisis de varianza (ANOVA) de dos factores (género y posición) con medidas repetidas para el segundo factor. La significación del análisis multivariado de medidas repetidas fue confirmada mediante los test Traza de Pillai, Lambda de Wilk, traza de Hotelling y raíz mayor de Roy, los cuales arrojaron resultados similares. La esfericidad fue analizada mediante la prueba de Mauchly. La corrección de Greenhouse-Geisser fue aplicada si la esfericidad no era asumida. Si se encontraban diferencias significativas en las variables dependientes para el efecto principal del ANOVA

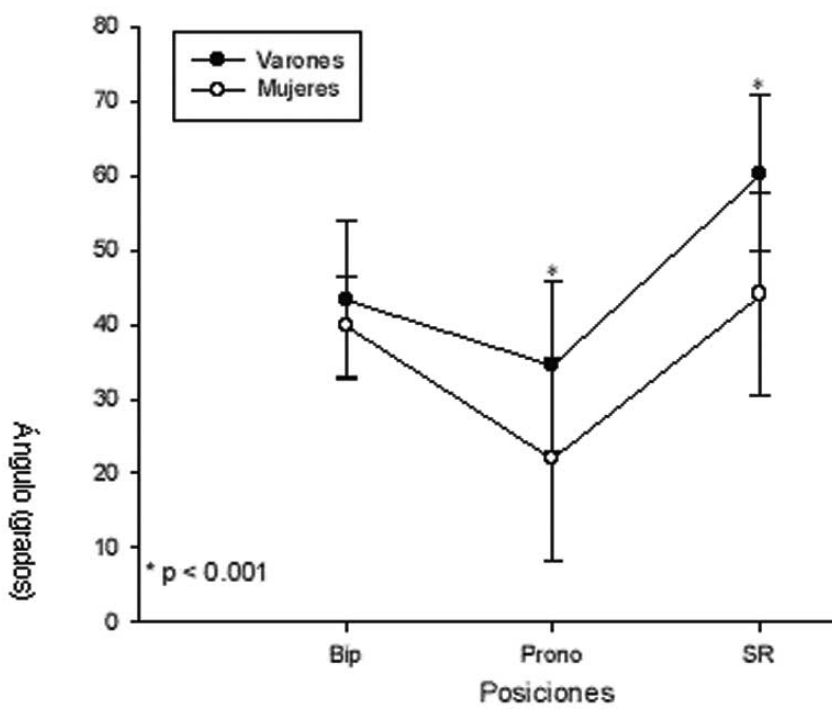

Fig. 3. Valores medios ( \pm desviación típica) de la cifosis torácica en las diferentes posiciones valoradas. $(\mathrm{p}<0,05)$, se procedió a realizar una comparación por pares (post hoc) usando la corrección de Bonferroni para comparaciones múltiples, ajustando el criterio de significación a un valor de 0,016 (0,05 dividido entre 3). Los datos fueron analizados usando el software SPSS (versión 15,0), y el nivel de significación se estableció en un valor de $\mathrm{p}<0,05$.

\section{RESULTADOS}

Los valores medios ( \pm desviación típica) de la cifosis torácica y curva lumbar en las diferentes posiciones analizadas, se presentan en las Figuras 3 y 4, respectivamente. El ANOVA mostró diferencias significativas para el efecto principal $(p<0,001)$ en los valores angulares de la curva torácica y lumbar. La interacción género x posiciones sólo evidenció diferencias significativas para la curva lumbar ( $\mathrm{p}<0,01)$. La cifosis torácica en los varones fue superior en las tres posiciones analizadas (Fig. 3). En la curva lumbar no se encontraron diferencias entre géneros en las posiciones evaluadas (Fig. 4). El análisis post hoc con ajuste de Bonferroni evidenció diferencias significativas en la cifosis torácica $(\mathrm{p}<0,016)$ en todas las comparaciones por pares, excepto entre las posiciones de bipedestación y el test sit-and-reach en las mujeres. En el raquis lumbar sólo hubo diferencias significativas entre el test sit-and-reach y las otras dos posiciones evaluadas. Tampoco hubo diferencias significativas entre las posturas de bipedestación y decúbito prono.

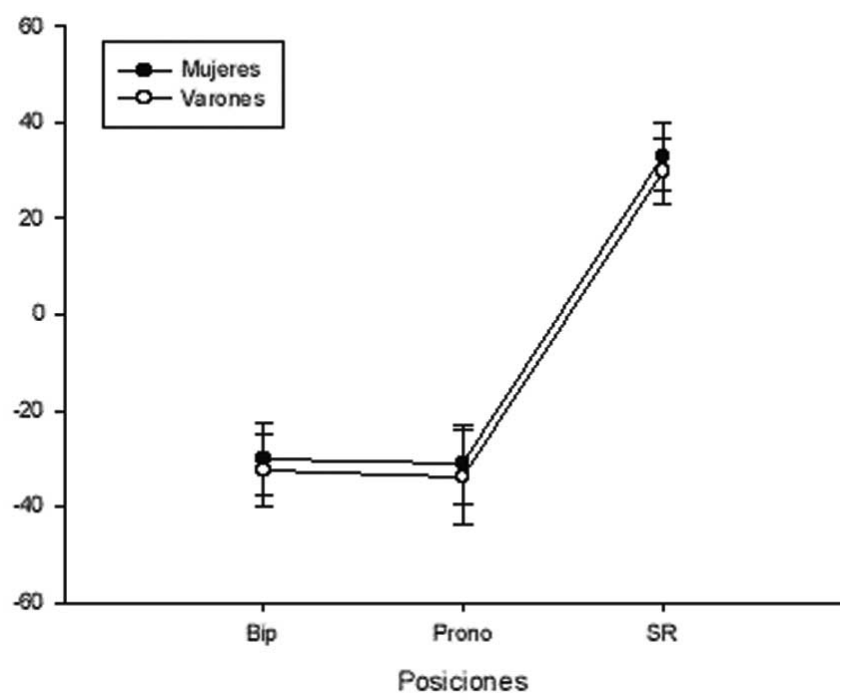

Fig. 4. Valores medios ( \pm desviación típica) de la curva lumbar en las diferentes posiciones valoradas. 
Al analizar los valores angulares en bipedestación en base a las referencias de normalidad, se encontró un mayor porcentaje de casos de hipercifosis torácica en los varones $(42,1 \%)$ que en las mujeres $(21,4 \%)$. Ningún deportista presentó una rectificación torácica. La cifosis torácica de los deportistas con hipercifosis fue significativamente menor en decúbito prono $\left(37,85 \pm 9,42^{\circ}\right)$ que en bipedestación $(50,95$ $\left.\pm 5,12^{\circ}\right)(\mathrm{p}<0,001)$. En decúbito prono, sólo el 10,9\% de los deportistas tenían una hipercifosis torácica (todos varones). En cuanto al raquis lumbar en bipedestación, predominaron las angulaciones consideradas normales $(81,6 \%$ en varones y $75,0 \%$ en mujeres). Un $10,5 \%$ de los varones y un $21,4 \%$ de las mujeres tenían una hiperlordosis lumbar, mientras que los casos de rectificación lumbar (hipolordosis) fueron más reducidos (7,9\% en varones y 3,6\% en mujeres). En decúbito prono, estos porcentajes fueron muy similares.

En el test sit-and-reach, el 26,3\% de los varones tenían un morfotipo cifótico en la curva torácica. Todas las mujeres tenían valores normales. En la curva lumbar, la mayoría de los deportistas presentaron morfotipos cifóticos leves $(36,8 \%$ en varones y $39,3 \%$ en mujeres) o moderados (55,3\% varones y $39,3 \%$ en mujeres).

\section{DISCUSIÓN}

Los principales hallazgos de este estudio fueron una relativa frecuencia de deportistas con hipercifosis torácica en bipedestación, especialmente en varones, así como una alta frecuencia de morfotipos cifóticos lumbares en el test sit-and-reach en ambos géneros. En bipedestación, las diferencias en la morfología raquídea entre géneros están relacionadas con una diferente geometría estructural entre varones y mujeres (Masharawi et al.). La mayoría de estas hipercifosis torácicas se caracterizaron por ser flexibles (no estructuradas), ya que en decúbito prono la cifosis torácica presentó una significativa reducción (unos $13^{\circ}$ de media). Estudios previos en escaladores (Föster et al.), luchadores (Rajabi et al.), esquiadores (Alricsson \& Werner), levantadores de pesas (López-Miñarro et al., 2009), así como en palistas de categoría infantil (López-Miñarro et al., 2009, 2010), han encontrado una alta frecuencia de casos con alteraciones raquídeas, si bien ninguno de estos estudios determinó si se trataba de desalineaciones estructuradas o posturales.

Los datos del presente estudio confirman la hipótesis de Fernández et al. que relacionaron la práctica del piragüismo/canotaje con una tendencia a la hipercifosis torácica. Esta postura aumenta el estrés compresivo y de cizalla, así como la presión intradiscal (Briggs et al.; Keller et al.; Wilke et al.; Polga et al.). Además, las posturas hipercifóticas alteran la cinemática de la articulación escápulo-humeral (Kebaetse et al., 1999) y reducen la eficacia ventilatoria (Landers et al., 2003), lo que puede suponer una disminución del rendimiento en estos deportistas.

En cuanto a la curva lumbar, Fernández et al., indicaron que la hiperlordosis es una desalineación frecuente en palistas de aguas tranquilas. El presente estudio mostró unos valores medios de lordosis lumbar que tienden a la normalidad, por lo que la hiperlordosis es una desalineación menos frecuente que la hipercifosis torácica, aunque en las mujeres el porcentaje fue mayor. La mayor frecuencia en las mujeres ha sido descrita previamente en estudios previos en población deportista y sedentaria (Masharawi et al.; LópezMiñarro et al., 2010).

La alta frecuencia de morfotipos cifóticos lumbares tanto leves como moderados en el test sit-and-reach, en ambos géneros, podría ser una adaptación a la posición de sedentación prolongada, con rodillas casi extendidas, de los kayakistas (López-Miñarro et al., 2009, 2010). Estos datos coinciden con Howell (1984), que encontró un alto porcentaje de hiperflexiones lumbares en remeras, posiblemente como una adaptación al gesto técnico cíclico del remo, que conlleva una significativa flexión lumbar. En el caso del kayakista, la postura del raquis lumbar en la posición específica de paleo en su piragua se caracteriza por una inversión lumbar, siendo mayor conforme más reducida es la extensibilidad isquiosural (López-Miñarro et al., 2010). Esta flexión mantenida podría generar una deformación viscoelástica de los tejidos ligamentosos del arco posterior de las vértebras (“creep deformation"), aumentando el rango de flexión intervertebral lumbar (Solomonow, 2004). Pastor, en nadadores, encontró una mayor frecuencia de acuñamientos vertebrales en la transición tóraco-lumbar en aquellos que alcanzaban una mayor flexión intervertebral en el test sit-and-reach. En el raquis torácico, sin embargo, la presencia de morfotipos cifóticos leves o moderados fue mucho más reducida, no existiendo caso alguno en las mujeres. Este hecho se explica porque una mayor extensibilidad isquiosural se relaciona con una menor cifosis torácica al realizar movimientos de flexión máxima del tronco con rodillas extendidas (López-Miñarro \& Alacid).

El seguimiento de la evolución de la postura corporal en deportistas debería integrarse en la planificación del entrenamiento, ya que las curvas raquídeas cambian con la edad (Cil et al.) y el entrenamiento sistematizado (Wojtys et $a l$.). Alricsson y Werner, en esquiadores de fondo, encontraron un incremento significativo de la cifosis torácica en bipedestación tras un período de 5 años de entrenamiento. Un adecuado análisis del morfotipo raquídeo permite detectar posibles desalineaciones raquídeas y, por tanto, diseñar un protocolo de tratamiento o de prevención adecuado. 
En conclusión, la morfología sagital del raquis en kayakistas de categoría cadete se caracteriza por una tendencia a la hipercifosis torácica, especialmente en los varones, si bien se trata de curvas no estructuradas. En cuanto a la curva lumbar en bipedestación existe un mayor porcentaje de ángulos normales. El piragüismo/canotaje en su modalidad de kayak genera un importante rango de flexión intervertebral lumbar, que deriva en un alto porcentaje de morfotipos cifóticos. Por ello, es preciso adoptar medidas de intervención postural para mejorar la morfología raquídea de los palistas de aguas tranquilas.

\section{AGRADECIMIENTOS}

Los autores agradecen su colaboración a la Real Federación Española de Piragüismo, así como a los entrenadores y deportistas que participaron en el estudio.

MUYOR, J. M.; ALACID, F.; LÓPEZ-MIÑARRO, P. A. Sagittal spinal morphology in young highly-trained paddlers. Int. J. Morphol., 29(3):1047-1053, 2011.

SUMMARY: The aim of this study was to determine the sagittal spinal morphology of thoracic and lumbar spine in young kayakers. A total of 66 young sprint kayakers, 36 males and 30 females (mean age: 16,22 $\pm 0,80$ ) participated in the study. Sagittal spinal curvatures of thoracic and lumbar spine in standing, lying prone and maximal trunk flexion with knees extended (sit-and-reach test) were measured with a Spinal Mouse. Thoracic kyphosis was higher in males than in females in all three analyzed postures, although greater differences were found in the sit-and-reach test. The frequency of thoracic hyperkyphosis was higher in males (42.1\%) than in females $(21.4 \%)$. The angular values when lying prone were more reduced than in standing $(\mathrm{p}<0.001)$. Paddlers with thoracic kyperkyphosis showed a reduced thoracic angle when lying prone $\left(37.85 \pm 9.42^{\circ}\right)$ than in standing $\left(50.95 \pm 5.12^{\circ}\right)$. No significant differences between sexes were found in the lumbar curve. A predominance of neutral angular values was found in the lumbar curve $(81.6 \%$ in males and $75.0 \%$ in females). In conclusion, the sagittal spinal morphology showed a trend toward thoracic hyperkyphosis in junior kayakers, specially in males. However, these curves were non-structural deformities. Lumbar spine showed a high percentage of neutral values. Kayak training is associated to improved lumbar intervertebral flexion and increased lumbar hyperkyphotic postures. It is necessary to include postural training to improve the spinal morphology of young paddlers.

KEY WORDS: Posture; Sagittal curvatures; Spine; Sport.

\section{REFERENCIAS BIBLIOGRÁFICAS}

Alricsson, M. \& Werner, S. Young elite cross-country skiers and low back pain. A 5-year study. Phys. Ther. Sport., 7(4):181-4, 2006.

Briggs, A.; Van Dieën, J.; Wrigley, T.; Greig, A.; Phillips, B.; Lo, S. \& Bennell, K. Thoracic kyphosis affects spinal loads and trunk muscle force. Phys. Ther., 87(5):595607, 2007.

Cil, A.; Yazici, M.; Uzumcugil, A.; Kandemir, U.; Alanay, A.; Alanay, Y.; Acaroglu, R.E. \& Surat, A. The evolution of sagittal segmental alignment of the spine during childhood. Spine, 30(1):93-100, 2005.

Fernández, B.; Terrados, N.; Pérez-Landaluce, J. \& Rodríguez, M. Patología del piragüismo. Arch. Med. Dep., 35:315-8, 1992.

Föster, R.; Penka, G.; Bösl, T. \& Schöffl, V. Climber's backform and mobility of the thoracolumbar spine leading to postural adaptations in male high ability rock climbers. Int. J. Sports Med., 30(1):53-9, 2009.
Howell, D. Musculoskeletal profile and incidence of musculoskeletal injuries in lightweight women rowers. Am. J. Sports Med., 12(4):278-81, 1984.

Kapandji, I. A. Physiologie articulaire. 1. Membre supérieur. 5è édition. Paris, Maloine, 1980.

Kebaetse, M.; McClure, P. \& Pratt, N. A. Thoracic position effect on shoulder range of motion, strength, and threedimensional scapular kinematics. Arch. Phys. Med. Rehabil., 80(8):945-50, 1999.

Keller, T. S.; Colloca, C. J.; Harrison, D. E.; Harrison, D. D. \& Janik, T. J. Influence of spine morphology on intervertebral disc loads and stresses in asymptomatic adults: implications for the ideal spine. Spine J., 5(3):297-300, 2005.

Kums, T.; Ereline, J.; Gapeyeva, H.; Pääsuke, M. \& Vain, A. Spinal curvature and trunk muscle tone in rhythmic gymnasts and untrained girls. J. Back. Musculoskelet. Rehabil., 20(2-3):87-95, 2007. 
Landers, M.; Barrer, G.; Wallentine, S.; McWhorter, J. W. \& Peel C. A comparison of tidal volume, breathing frequency, and minute ventilation between two sitting postures in healthy adults. Physiother. Theory Pract., 19(2):109-19, 2003.

López-Miñarro, P. A. \& Alacid, F. Influence of hamstring muscle extensibility on spinal curvatures in young athletes. Sci. Sports, 25(4):188-93, 2010.

López-Miñarro, P. A.; Alacid, F. \& Muyor, J. M. Comparación del morfotipo raquídeo y extensibilidad isquiosural entre piragüistas y corredores. Rev. Int. Med. Cienc. Act., 9(36):379-92, 2009.

López-Miñarro, P. A.; Alacid, F. \& Rodríguez-García, P. L. Comparison of sagittal spinal curvatures and hamstring muscle extensibility among young elite paddlers and non-athletes. Int. Sport Med. J., 11(2):301-12, 2010.

López-Miñarro, P. A.; Alacid, F.; Ferragut, C. \& García, A. Valoración y comparación de la disposición sagital del raquis entre canoístas y kayakistas de categoría infantil. Cult. Cien. Dep., 3(9):171-6, 2008.

Masharawi, Y.; Dar, G.; Peleg, S.; Steinberg, N.; Medlej, B.; May, H.; Abbas, J. \& Hershkovitz, I. A morphological adaptation of the thoracic and lumbar vertebrae to lumbar hyperlordosis in young and adult females. Eur. Spine J., 19(5):768-73, 2010.

Mejia, E. A.; Hennrikus, W. L.; Schwend, R. M. \& Emans, J. B. A prospective evaluation of idiopathic left thoracic scoliosis with MRI. J. Pediatr. Orthop., 16(3):354-8, 1996.

Nilsson, C.; Wykman, A. \& Leanderson, J. Spinal Sagittal mobility and joint laxity in young ballet dancers. Knee. Surg. Sports Traumatol. Arthroscopy, 1(3-4):206-8, 1993.

Pastor, A. Estudio del morfotipo sagital de la columna y de la extensibilidad de la musculatura isquiosural de jóvenes nadadores de élite Españoles. Tesis Doctoral. Murcia, Universidad de Murcia, 2000.

Polga, D. J.; Beaubien, B. P.; Kallemeier, P. M.; Schellhas, K. P.; Lee, W. D.; Buttermann, G. R. \& Wood, K. B. Measurement of in vivo intradiscal pressure in healthy thoracic intervertebral discs. Spine, 29(12):1320-4, 2004.

Rajabi, R.; Doherty, P.; Goodarzi, M. \& Hemayattalab, R.
Comparison of thoracic kyphosis in two groups of elite Greco-Roman and free style wrestlers and a group of non-athletic subjects. Br. J. Sports Med., 42(3):229-32, 2008 .

Smith, A.; O’Sullivan, P. \& Strajer, L. Classification of sagittal thoraco-lumbo-pelvic alignment of the adolescence spine in standing and its relationship to low backpain. Spine, 33(19): 2101-7, 2008.

Solomonow M. Ligaments: a source of work-related musculoskeletal disorders. J. Electromyogr. Kinesiol., 14(1):49-60, 2004.

Tüzün, C.; Yorulmaz, I.; Cindas, , A. \& Vatan, S. Low back pain and posture. Clin. Rheumatol., 18(4):308-12, 1999.

Wilke, H.; Neef, P.; Caimi, M.; Hoogland, T. \& Claes, L. New in vivo measurements of pressures in the intervertebral disc in daily life. Spine, 24(8):755-62, 1999.

Wojtys, E.; Ashton-Miller, J.; Huston, L. \& Moga, P. The association between athletic training time and sagittal curvature of the immature spine. Am. J. Sports Med., 28(4):490-8, 2000.

Dirección para correspondencia:

Pedro A. López-Miñarro

Department of Physical Education.

Faculty of Education. Campus Universitario de Espinardo. CP. 30100 Murcia

ESPAÑA

Telephone: 34868887051

Fax: 34868884146

Email: palopez@um.es

Recibido : 18-01-2011

Aceptado: 31-03-2011 